\title{
A Robust State Estimator Based on Maximum Constraints Satisfaction of Uncertain Measurements
}

\author{
A.K. AL-Othman \\ Electrical Engineering Department \\ Collage of Technological Studies \\ Al-Rawda, 73452, P.O. Box 33198, Kuwait
}

\author{
M.R. Irving \\ Brunel Institute of Power Systems \\ Brunel University \\ Uxbridge, Middlesex UB8 3PH, UK
}

\begin{abstract}
'Abstract: - A new robust estimator based on the concept of uncertainty in the measurements is developed in this paper. The uncertainty in the measurements is modeled via deterministic upper and lower bounds on measurement errors, which take into account known meter accuracies. Inequality constraints are constructed to model the uncertainty in the measurements. A solution point satisfying most inequality constraints is the objective of the proposed estimator. Hence, this estimator is known as Maximum Constraints Satisfaction (MCS). The Robustness and performance of the proposed estimator is discussed via simulated problems of simple regression examples and D.C. three-bus system. Various scenarios of leverage measurements and bad data have been considered for further assessment of the performance of the MCS estimator. In particular, it is shown that the (MCS) estimator performs significantly well in situation where collinearity exists in the measurements. Results show that the proposed estimator is an accurate and reliable estimator.
\end{abstract}

Key-Words: - Robust State Estimation, Genetic Algorithms (GA), Measurements Uncertainty.

\section{Introduction}

In traditional power system state estimation, estimators based on statistical methods such as least squares and least absolute values, along with their variants, are well documented and have been widely applied. Researchers have realized that measurements acquired from the field usually do not entirely satisfy the assumptions on which those estimators have been initially built upon. Also, circumstances such as faulty measurements pose a serious threat to the quality of the estimator outcome. Hence, robust estimators have been introduced as an alternative, to eliminate or down-weigh the effects of faulty measurements (or outliers). Normally, outliers occur quite frequently, as a consequence of high amplitude noise in measurements (e.g., due to induction during large transients), brief loss of measurement data and noise occurring due to unintended signal paths and measurements [1]. Furthermore, many AC applications result in frequent measurement errors and "periodic noise". Periodic errors are common in power electronic switched applications.

Generally, robust estimators are those estimators that exhibit stable behavior (bounded bias and variance) under deviation from the assumptions on which they are based. That way, any outliers would be identified and eliminated from the measurement set, prior to the estimation process. An alternative definition of robust estimators is that they are those estimators that are resistant against all kinds of outlier and leverage points.

When the LMS estimator was initially appealing due to its robustness and effectiveness in detecting outliers. Unfortunately, LMS suffers from poor performance in terms of asymptotic efficiency. In fact the LMS has an asymptotic efficiency of zero [2], which means that the LMS has an apparently slow convergence rate. Furthermore, a situation where sever problems can potentially occur for LMS is when collinearity exists in the measurements. As stefanski has argued that high-breakdown estimators, e.g. LMS, can exhibit unusual finite-sample behavior [3]. Stefanski has shown, via a simple example, that an LMS estimator may produce dubious estimates where collinearity does exist among a certain set of measurements. This simple example was slightly modified by Ryan, in [4], to further illustrate the weakness of the LMS estimator. The outcome of LMS and LS estimators on the Stefanski example is illustrated in Fig. 1. It appears from Fig.1 that the outliers have successfully misled the LMS fit and attracted its line. This phenomenon is mainly caused by collinearity in the measurements. In this example $m=9$ and $n=1$, therefore the 5 lower valued squared residuals will be minimized by LMS. Interestingly, five points of the data set fall on the same line, three good points and two outliers, which consequently 
appears to be the best candidate solution that the can be provided by the LMS estimator.

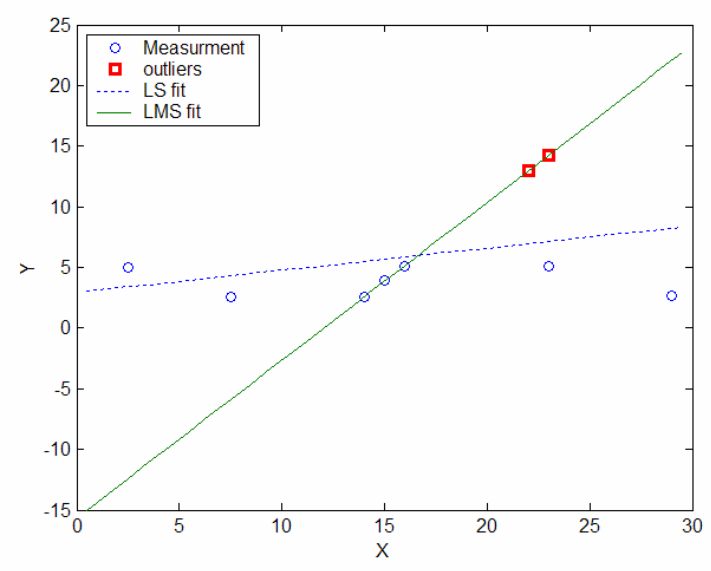

Figure1. Illustration of LMS shortcoming

Thus, for this example, the LMS solution was very far from the correct solution. In comparison, the outliers seem to have less effect on the LS estimate (which a non robust estimator). Subjectively, the LS fit appears to be better and closer to a correct solution (a correct solution should have approximately a zero slope), even though it is none-robust and is known to have $0 \%$ breakdown point [5]). Certainly, had the seven good data only been used in the LMS regression procedure, the slope would have been virtually zero. However, the collinearity of three good data points with two outliers has misled the LMS estimator. Despite that, LMS and the LTS is still one of the most common robust regression estimators that is frequently used.

\section{Problem Formulation}

\subsection{Uncertainty and state estimation}

Uncertainty in power system state estimation is mainly due to measurement inaccuracy and network mathematical model used. For instance meters inaccuracies, communication error are major sources of measurements uncertainty. Parameters approximations in modeling of the Pi-equivalent, such as line resistance, reactance, hunt capacitance etc, also contribute to the uncertainty in state estimation. Unfortunately, magnitudes of such errors and approximations are not known, which in turn lead to uncertainty in the estimates obtained in state estimation. Schweppe [6] introduced the concepts of uncertainty in the general context of engineering analysis, estimation and optimization. These concepts have been extended and developed recently and have been applied in a number of areas. Uncertainty estimation has been considered in the context of water distribution networks. Bargiela and
Hainsworth [7] introduced bounds on the measurements, with an intention to increase the robustness of estimation. The approach was developed by Brdys and Chen [7], who introduced the term Set Bounded State Estimation (SBSE). Nagar and Powell [8] apply concepts from robust control theory and allowed for uncertainty in both the parameters and the measurements. The uncertainty is isolated with the use of a Linear Fractional Transformation (LFT), and the problem is formulated as a convex semi-definite programming (SDP) problem. A Linear Matrix Inequalities (LMI) approach is then used to solve the SDP. Al-Othman and Irving presented in [9] a method for estimating the uncertainty interval around the system state variables in power system. The method is based on using WLS and linear programming to find the upper and lower bounds on the estimated states. The uncertainty however is modeled only in the measurements. One limitation with this method is that it relies upon a static estimator, i.e. WLS, which performs well in the presence of unbiased normally distributed errors in the measurements. Therefore, the (LP) solution might be compromised, if the measurements were contaminated with outliers or unexpected error distributions are encountered.

\subsection{The Maximum Constraints Satisfaction Estimator}

The concept of uncertainties in the measurements may be considered to develop a more robust estimator. In this section a new robust state estimator is proposed based on measurements uncertainty. The uncertainty is modeled via deterministic upper and lower bounds on measurement errors, which take into account known meter accuracies. Inequality constraints are constructed of the given measurements to model the uncertainty in the measurements. A solution point satisfying most inequality constraints is the objective of the proposed estimator.

In power system state estimation, inequality constraints are usually needed in optimization to deal with uncertainties. In [10], an inequality constraints is employed, in a LVA estimator, only for handling uncertainty in pseudomeasurements since they are not measured but they are known to vary within bounded interval. An inequality constraints LAV estimator based on penalty functions was formulated, in [11], to estimate states of external systems. A parameter-bounding model derived from the bounded noise measurements was used in [12] with a reformulated constrained WLS, to handle unmeasured loads in the system. The proposed MCS 
estimator is based on the idea of searching for a point $\underline{x}$ in the space of all possible estimates that maximizes the number of satisfied uncertain measurements. To be specific, each measurement with its associated uncertainty can be represented by double inequality constraints (upper and lower limits). These constraint limits define the tolerances on the measurements (i.e. the range of values within which the true value of the measured quantity must lie). A point $\underline{x}$ satisfying most of the available double inequality constraint, if not all, must be a valid solution point. The fundamental concept of the MCS estimator may be explained better by the illustration in Fig. 2. This illustration shows three uncertain measurements along with their bounds (upper and lower limits). The intersection of these bounds defines an area known as the "feasible region" of the uncertain measurements. An arbitrary point in the feasible region may certainly be considered as a potential solution that satisfies all those uncertain measurements (i.e. double inequality constraints). It must be noted that any given solution point in the feasible region is not necessarily optimum but is a valid feasible solution. Suppose that outliers exist in the measurement set. These outliers with their bounds might, or might not, create a region of their own. If by coincidence the uncertain bounds of the erroneous measurements mange to establish a region of their own, a solution point in that region can never have a maximum number of satisfied constraints that exceeds that of the region established by the good measurements, (given that the level of contamination in a given set of measurements can never be more than $50 \%$, for the worst case scenario). Therefore, the MCS estimator guarantees a robust solution, which is not influenced by outliers or erroneous measurements. That is because the MCS estimator always seeks a solution point in the feasible region with the largest number of satisfied measurements, (which ought to be created only by the good measurements in the set). Consequently, existence of outliers or erroneous measurements can never affect the quality of the MCS solution.

Mathematically, for a vector of measurements $\underline{Z}$, the uncertain measurements may be represented by the following double inequality constraints:

$$
\begin{gathered}
z_{1}^{l} \leq h(\underline{x}) \leq z_{1}^{u} \\
\vdots \quad \vdots \quad \vdots \\
z_{m}^{l} \leq h(\underline{x}) \leq z_{m}^{u}
\end{gathered}
$$

where $h(\underline{x})$ is set of mathematical equation that relates the states $\underline{X}$ to the measurements $\underline{Z}$. Also $\underline{Z}^{l}$ is the lower bound of the measurement vector and $\underline{z}^{u}$ is upper bound, and they are formed as:

$$
\begin{aligned}
& \underline{z}^{l}=\underline{z}-\underline{\tau}^{-} \\
& \underline{z}^{l}=\underline{z}-\underline{\tau}^{+}
\end{aligned}
$$

where $\underline{\tau}^{+}$and $\underline{\tau}^{-}$are the transducer tolerances. The tolerance describes the deterministic uncertainty of each measurement. It represents the overall accuracy of the meter and can usually be provided by the manufacturer. Different values for the elements of positive and negative tolerances are permissible so that a transducer can be specified to have asymmetric accuracy if required (e.g. an accuracy of $-3 \%$ to $+5 \%$ of the nominal value). Nevertheless, without loss of generality, we will usually assume that $\underline{\tau}^{+}=\underline{\tau}^{-}=\underline{\tau}$, giving a symmetric tolerance around the nominal value.

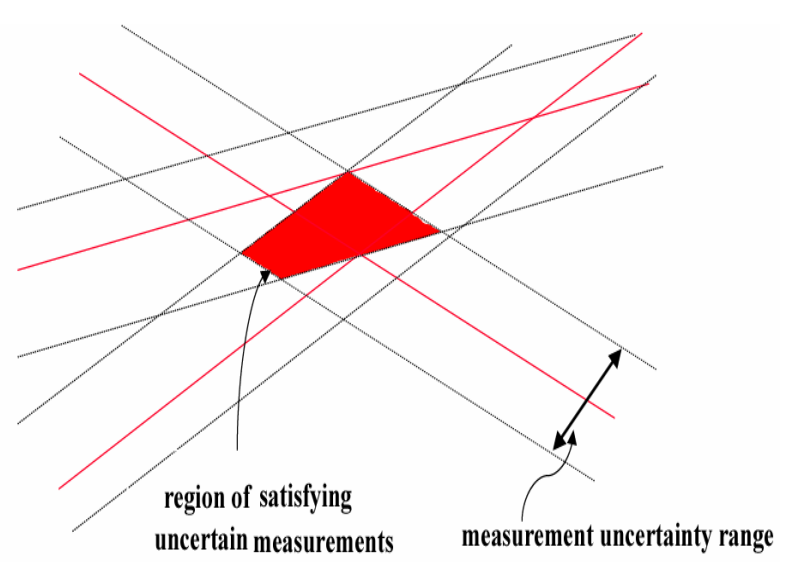

Figure2. The Maximum Constraints Satisfaction The proposed estimator aims at searching for a particular state variable vector that maximizes the number of satisfied uncertain measurements (double inequality constraints) described in equation (1). Formally the objective function of the proposed estimator is to maximize the number of satisfied constraints for a given potential solution $\underline{x}$.

$$
\operatorname{Max} \Omega
$$

where $\Omega$ denotes the number of double inequality constraints of equ. (1) satisfied by $\underline{x}$. Geometrically, the shape of the objective function may be analogous to a campus map, where the objective is to search for the top of the tallest building. The value $\Omega$ is analogous to the number of floors in each building, and $\underline{x}$ represents the ground co-ordinates of a point on the campus. The tallest top is the feasible region established by the good measurements. For that reason, a Real-Coded Genetic Algorithm is chosen to solve the 
optimization problem of equation 4, which starts by generating a random population of potential solutions in the real space, of which each potential solution is evaluated and checked for how many double inequality constraints are satisfied. The potential solution with highest number of satisfied constraints, $\Omega_{i}$, is picked up by RGA for the next generation, and so on. Additionally, Genetic Algorithm was chosen because of its capability to search the whole space to find that region with the most satisfied constraints. In the following section the proposed method has been tested on simple regression test cases.

\section{Problem Solution}

\subsection{Simple linear regression example}

For a simple regression test case, with $m$ measurements, the MCS general formulation may be given as:

$$
\begin{gathered}
\operatorname{Max}_{\theta_{1}, \theta_{2}} \Omega \\
z_{1}^{l} \leq x_{11} \theta_{1}+\theta_{2} \leq z_{1}^{u} \\
\vdots \quad \vdots \quad \vdots \quad \vdots \quad \text { where } m \succ 2 \\
z_{m}^{l} \leq x_{m 1} \theta_{1}+\theta_{2} \leq z_{m}^{u}
\end{gathered}
$$

Where $\theta_{1}$ and $\theta_{2}$ are the regression coefficients to be estimated. We can now apply the MCS estimator on an example which had two outliers in the $x$-direction from section. It was demonstrated in [2], that the LMS estimator has successfully detected these two outliers. With a symmetric tolerance of, $\tau= \pm 3 \%$, applied on all seven measurements of that example, the MCS formulation would be

$$
\begin{gathered}
\operatorname{Max}_{\theta_{1}, \theta_{2}} \Omega \\
z_{1}^{l} \leq x_{1} \theta_{1}+\theta_{2} \leq z_{1}^{u} \\
\vdots \quad \vdots \quad \vdots \quad \vdots \\
z_{7}^{l} \leq x_{7} \theta_{1}+\theta_{2} \leq z_{7}^{u}
\end{gathered}
$$

The MCS has effectively solved for the correct regression coefficients, $\hat{\theta}_{\mathrm{MCS}}=\left[\begin{array}{c}0.5 \\ 0\end{array}\right]$, fitting all five good points, perfectly unaffected by the outliers in the $x$-direction. The least squares fit is shown for comparison in figure 3 . The bottom subfigure in fig. 3 , illustrates the number of satisfied constraints, $\Omega$, progressively increasing throughout the evolution process of GA. It is interesting to see that the maximum number of constraints that could possibly be satisfied is five, in this case, which is the number of good measurements.
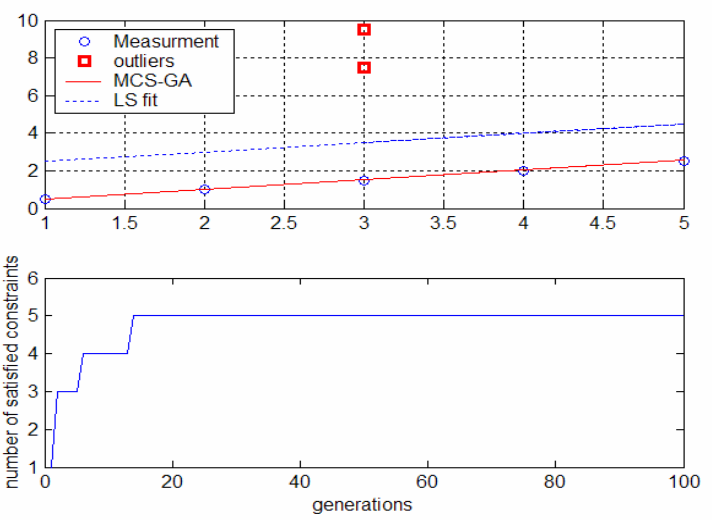

Figure3. Application of MCS with outliers in the $x$-direction

As expected, when the MCS estimator has been applied to a simple regression case which had two outliers in the $y$-direction, both leverage points, the regression parameters were $\hat{\theta}_{\mathrm{MCS}}=\left[\begin{array}{c}0.5 \\ 0\end{array}\right]$. The outcome of the MCS is compared with least squares, and an illustration of that outcome is shown in Fig 4. Clearly, these two leverage points had no effect on the MCS estimator. (For these tests, the population size $=100$, crossover $=0.8$, mutation $=0.09$ and elitism rate $=8 \%$.)

For further assessment of the robustness and performance of the MCS estimator has been tested in situations where collinearity exists, the MCS estimator has also been tested on the data set provided by Stefanski [3]. (With tolerance $\tau= \pm 3 \%$, population size $=100$, crossover $=0.8$, mutation $=0.09$ and elitism rate $=8 \%$ ). Fig. 5 illustrates the outcome of the MCS estimator on Stefanski's example. Apparently, the solution from that run has produced a zero slope approximately, to be specific $\hat{\theta}_{\mathrm{MCS}}=\left[\begin{array}{c}-0.0017 \\ 3.4088\end{array}\right]$.
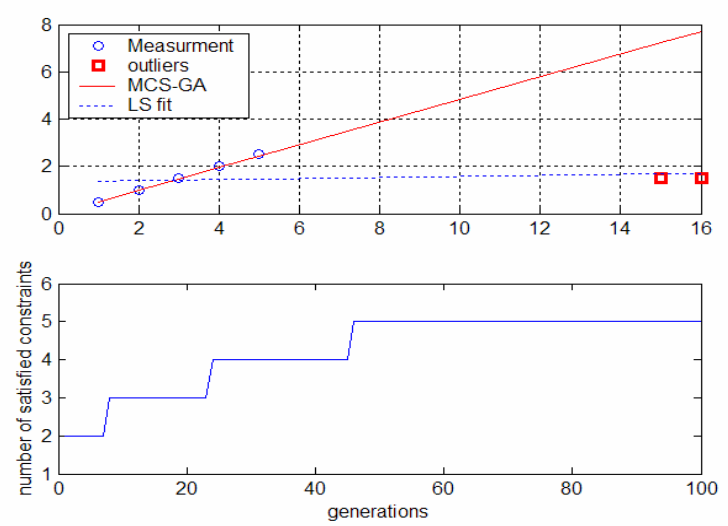

Figure4. Application of MCS with outliers in the $y$-direction 
As far as the maximum number of satisfied measurements is concerned, it is apparent form the plot that only four double inequality constraints had been satisfied in the specific run; giving four data points adjacent to the MCS curve in the figure. This suggests that the number of satisfied constraints cannot always be used as an indication of number of outliers in the measurements. Had the tolerance been wider, (e.g. $\tau= \pm 5 \%$ ), more measurements would have been satisfied. In this example, outliers could be visually identified, however for higher dimension problems, residual analysis may be carried out for proper detection of the number of outliers.
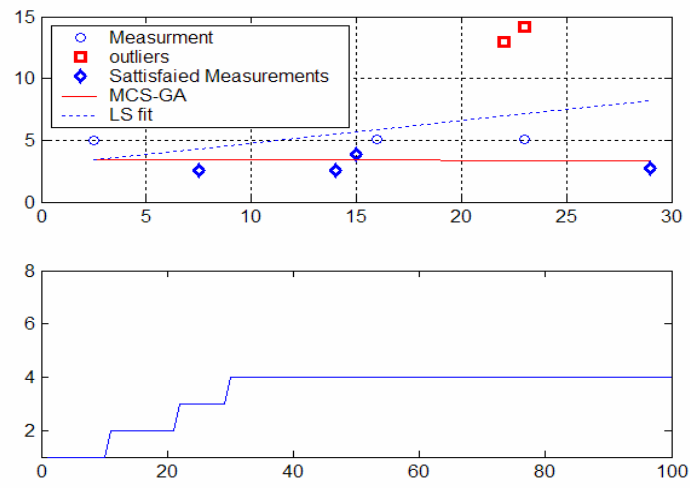

Figure 5. Application of MCS on Stefanski’s example 3.2 D.C. Three bus test system

For further assessment and testing of the MCS estimator on a more realistic example, the D.C. model of a three-bus system is considered. An on-line diagram of the test system is displayed in Fig. 6 . The system was originally proposed by Monticelli and et. al, in [13], in their study of the identification of multiple bad data for state estimation. Milli et. al, in [14], have applied the LMS estimator on the same system. Physically, the resistance of all lines of the three-bus network are taken to be zero. All line reactances are however to 0.1 p.u. Suppose that the six real power measurements are taken with zero variances, the measurements Jacobian is given as:

$$
H=\left[\begin{array}{cccccc}
10 & -10 & 10 & 0 & -10 & -10 \\
-10 & 10 & 0 & 10 & 5 & -10
\end{array}\right]^{T}
$$

Having assumed bus1 as the slack, (reference bus), the true states $\theta_{2}$ and $\theta_{3}$ are zero. Hence, the true flows and injections are all zero, i.e. $Z=\left[\begin{array}{llllll}0 & 0 & 0 & 0 & 0 & 0\end{array}\right]$. In order to test the MCS estimator, various scenarios of bad data occurring in the measurement set will be considered. For the sack of checking the validity of the proposed method, these scenarios are exactly the same as those considered in [13].

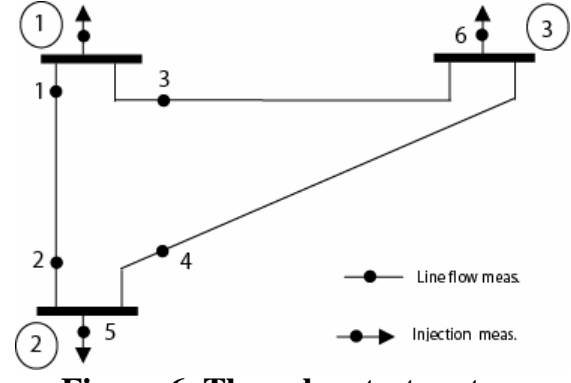

Figure 6. Three-bus test system

Case A: Suppose that a single bad data had been acquired for the fifth measurement, the real power injection at bus 2, for example $Z=\left[\begin{array}{llllll}0 & 0 & 0 & 0 & 1 & 0\end{array}\right]$. The MCS estimator yields $\theta_{2}=0.0010$ and $\theta_{3}=0.0008$. From that solution the residual is calculated as [ $-0.0027, \quad 0.0027, \quad-0.0102, \quad-0.0075, \quad 1.0065$, 0.0177], and the corresponding standard deviation for the residuals is 0.4110 . It is apparent that the fifth residual is the only one which is larger than the standard deviation, suggesting that the fifth measurement must be a bad data point.

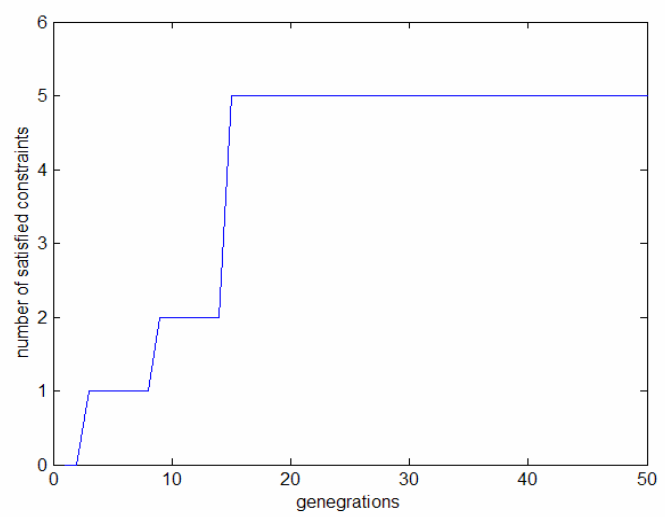

Figure 7. MCS performance with single bad data

Figure 7, displays the convergence of the MCS estimator. It shows that only five double inequality constraints can possibly be satisfied in this case, signifying that one measurement in the set must be erroneous.

Case B: Assume that readings for the same network

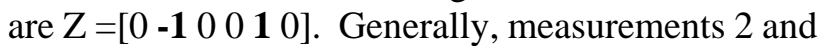
5 are considered interacting, since they are both related to the line flows of bus2. These measurement values are however nonconforming. The MCS estimator yields $\theta_{2}=-0.0039$ and $\theta_{3}=0.0132$, leading to residual vector $[-0.0274,-\mathbf{- 0 . 9 7 2 6}$, $-0.0129, \quad 0.0145, \mathbf{1 . 0 2 1},-0.0016]$ and a standard deviation 0.6305 . From this result, it can be deduced that measurement 2 and 5 are erroneous. This type of error distribution is usually known as multiple interacting nonconforming bad data.

The maximum number of constraints that may be satisfied is four, as indicated by fig.8. That indication 
appears to be correct, since it is known that two out of the six readings are bad.

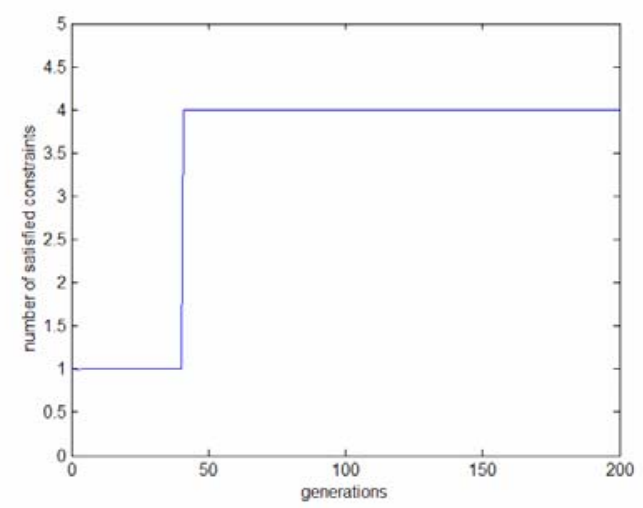

Figure 8. MCS performance with multiple interacting bad data

\section{Conclusion}

In this paper a robust estimator based on the satisfaction of uncertain measurements, has been presented. The robustness of the MCS has been successfully demonstrated through different simple regression examples and a three-bus D.C.. Various scenarios of leverage measurements and bad data have been considered for further assessment of the performance of the MCS estimator. Specifically the MCS estimator has been very effective as apposed to LMS, in situations where collinearity exists amongst measurements, in the case of linear regression. The distinct robustness of the MCS may be due to the uncertainty tolerance on the measurements, such that no exact fit of measurements is required by the proposed method. For accurate estimation, the use of the MCS estimator is recommended for identification and elimination of the outliers, prior the use of any non-robust estimator.

\section{References:}

[1]N. Logic, E. Kyriakides, and G. T. Heydt, "Lp State Estimators for Power Systems," Journal of Electric Power Components and Systems, accepted for publication, 2002.

[2] P. J. Rousseeuw and A. M. Leroy, Robust regression and outlier detection. New York ; Chichester: Wiley, 1987.

[3]A. Stefanski, "A note on HighBreakdown Estimator," Statistics and Probability, vol. 11, pp. 353-358, 1991.

[4]P. Ryan Thomas, Modern regression methods. New York ; Chichester: J. Wiley, 1997.

[5]P. J. Rousseeuw and V. J. Yohai, "Robust regression by means of Sestimators," in Robust and Nonlinear Time Series Analysis, Franke, J.,
HKardle, W., Martin, R.D. (Eds.), Lecture Notes in Statistics, vol. 26. New York: pp. 256-272,Springer, 1984.

[6]F. C. Schweppe, Uncertain dynamic systems. Englewood Cliffs, N.J.,: Prentice-Hall, 1973.

[7]A. Bargiela and G. Hainsworth, "Pressure and flow uncertainty in water system," ASC J. Water Resources Planning Mang, vol. 115 (2), pp. 212 229, 1989.

[8]A. Nagar and R. Powell, "LFT/SDP approach to the uncertainty analysis for state estimation of water distribution systems," IEE Proceedings on. Control Theory and Applications, vol. 149, pp. 137 -142, Mar 2002.

[9]A. K. AL-Othman and M. R. Irving, "Uncertainty Modeling in Power System State Estimation," Submitted for review to Generation, Transmission and Distribution, IEE Proceedings-, 2003.

[10]A. Abur and M. K. Celik, "Least absolute value state estimation with equality and inequality constraints," Power Systems, IEEE Transactions on, vol. 8, pp. 680 - 686, May 1993.

[11]H. Singh, F. L. Alvarado, and W.-H. E. Liu, "Constrained LAV state estimation using penalty functions," Power Systems, IEEE Transactions on, vol. 12, pp. 383 - 388, Feb. 1997.

[12]K. A. Clements, P. W. Davis, and K. D. Frey, "Treatment of inequality constraints in power system state estimation," Power Systems, IEEE Transactions on, vol. 10, pp. 567 - 574, May 1995.

[13]A. Monticelli, F. Wu, and M. Yen, "Multiple bad data identification for state estimation by combinatorial optimisation," Power Delivery, IEEE Transactions on, vol. PWRD-1, No.3, pp. 361-369, 1996.

[14]L. Mili, V. Phaniraj, and P. J. Rousseeuw, "Least median of squares estimation in power systems," Power Systems, IEEE Transactions on, vol. 6, pp. $511-523,1991$. 